\title{
Zero-dimensional lumped approach to incorporate the dynamic part of the pressure at vessel junctions in a 1D wave propagation model
}

\author{
Citation for published version (APA): \\ van den Boom, T., Stevens, R., Delhaas, T., van de Vosse, F., \& Huberts, W. (2018). Zero-dimensional lumped \\ approach to incorporate the dynamic part of the pressure at vessel junctions in a 1D wave propagation model. \\ International Journal for Numerical Methods in Biomedical Engineering, 34(9), [e3116]. \\ https://doi.org/10.1002/cnm.3116
}

DOI:

10.1002/cnm.3116

Document status and date:

Published: 01/09/2018

\section{Document Version:}

Accepted manuscript including changes made at the peer-review stage

\section{Please check the document version of this publication:}

- A submitted manuscript is the version of the article upon submission and before peer-review. There can be important differences between the submitted version and the official published version of record. People interested in the research are advised to contact the author for the final version of the publication, or visit the $\mathrm{DOI}$ to the publisher's website.

- The final author version and the galley proof are versions of the publication after peer review.

- The final published version features the final layout of the paper including the volume, issue and page numbers.

Link to publication

\footnotetext{
General rights

- You may freely distribute the URL identifying the publication in the public portal. follow below link for the End User Agreement:

www.tue.nl/taverne

\section{Take down policy}

If you believe that this document breaches copyright please contact us at:

openaccess@tue.nl

providing details and we will investigate your claim.
}

Copyright and moral rights for the publications made accessible in the public portal are retained by the authors and/or other copyright owners and it is a condition of accessing publications that users recognise and abide by the legal requirements associated with these rights.

- Users may download and print one copy of any publication from the public portal for the purpose of private study or research.

- You may not further distribute the material or use it for any profit-making activity or commercial gain

If the publication is distributed under the terms of Article 25fa of the Dutch Copyright Act, indicated by the "Taverne" license above, please 


\title{
Zero-dimensional lumped approach to incorporate the dynamic part of the pressure at vessel junctions in a 1D wave propagation model
}

\author{
Tim van den Boom ${ }^{1}$ (D) | Raoul Stevens ${ }^{2,3} \mid$ Tammo Delhaas $^{2,3} \mid$ Frans van de Vosse $^{1,3}$ | \\ Wouter Huberts ${ }^{1,3}$
}

${ }^{1}$ Department of Biomedical Engineering, Eindhoven University of Technology,

Eindhoven, The Netherlands

${ }^{2}$ Department of Biomedical Engineering, School for Mental Health and

Neuroscience (MHeNs), Faculty of Health, Medicine and Life Sciences, Maastricht

University, Maastricht, The Netherlands

${ }^{3}$ Department of Biomedical Engineering, School of Cardiovascular Diseases (CARIM), Faculty of Health, Medicine and Life Sciences, Maastricht University,

Maastricht, The Netherlands

\section{Correspondence}

Wouter Huberts, PO Box 616, UNS50

R3.354, 6200 MD Maastricht.

Email:

wouter.huberts@maastrichtuniversity.nl

\begin{abstract}
A benchmark study by Boileau et al tested 6 commonly used numerical schemes for 1D wave propagation, for their ability to capture the main features of pressure, flow, and area waveforms in large arteries. While all numerical schemes showed good agreement in pressure and flow waveforms for smaller arterial networks, the simplified trapezium rule method proposed by Kroon et al showed an overestimation for the systolic pressure of $1 \%$ in proximal regions and an underestimation of $3 \%$ in distal regions in comparison with the 5 other schemes when using a larger arterial network, published as the ADAN56 model. The authors attributed this difference to the neglection of the dynamic part of the pressure at vessel junctions. Carson et al resolved these differences by proposing 2 methods to implement the dynamic part of the pressure in the simplified trapezium rule method scheme. In the present study, an alternative method is introduced extending the work by Kroon et al. This alternative method consists of a new 0D element, which is placed at vessel junctions. The strength of this new element is the ease of implementation and its flexible coupling with other elements, without introducing additional degrees of freedom or the need of a penalty function. This new approach is compared with 5 other numerical schemes, which already have the dynamic part of the pressure incorporated. The new method shows excellent agreement with these schemes for the ADAN56 model.
\end{abstract}

\section{KEYWORDS}

arterial network, dynamic pressure, junctions, lumped approach, 1D wave propagation

\section{1 | INTRODUCTION}

One-dimensional (1D) network models have extensively been used to investigate blood pressure and blood flow wave propagation phenomena in arteries and veins. ${ }^{1-3}$ These so-called pulse wave propagation models allow the assessment of the effects of vascular disease on the pressure and flow waveforms, wave reflections, and the relation between central and peripheral pressures. The ease of use and the relatively low costs make 1D models also attractive for vascular intervention planning ${ }^{4}$ or for estimating boundary conditions for higher dimensional models. ${ }^{5}$ 
These 1D network models consist of connected elements that locally describe the relation between pressure, area, and flow. The pressure and flow relations are based on the $1 \mathrm{D}$ mass and momentum equations. ${ }^{6}$ At terminal branches, the 1D network is often truncated with, for example, 0D windkessel elements ${ }^{4}$ or structured outflow trees. ${ }^{7}$ To solve the governing equations derived from $0 \mathrm{D}$ and $1 \mathrm{D}$ models, different mathematical formulations and corresponding numerical methods have been proposed. These methods vary from each other by using different formulations of the 1D equations and different constitutive laws to relate area and pressure. In addition, they differ with respect to the velocity profiles used to estimate the friction and convective terms, choice in boundary conditions, the way of coupling at junctions, and the numerical schemes. ${ }^{8}$

Recently, Boileau et $\mathrm{al}^{9}$ compared 6 numerical schemes that are commonly used for 1D arterial blood flow modeling to investigate the effect of different numerical implementations on the pressure and flow waveforms in several benchmark problems while using the same velocity profile, boundary conditions, and constitutive laws. ${ }^{9}$ The numerical schemes considered were locally conservative Galerkin, ${ }^{10}$ discontinuous Galerkin, ${ }^{11}$ Galerkin least-squares finite element method $^{12}$ (FEM), finite volume method, ${ }^{13}$ MacCormack finite difference method, ${ }^{14}$ and the simplified trapezium rule method ${ }^{15}$ (STM). The results showed good agreement among all numerical schemes for 6 benchmark problems (ie, a reflection free tube, a tube mimicking the common carotid artery, the upper thoracic aorta, an aortic bifurcation, and experimental arterial networks with 37 and 56 of the larger arteries, published as the ADAN56 model). It was concluded that all schemes are able to solve nonlinear 1D equations and to capture the main features of pulse wave propagation. However, for the ADAN56 model, noticeable discrepancies were obtained when using the STM in comparison with the other numerical schemes. While the STM showed to be able to solve the system of equations within minutes, ${ }^{9,15}$ systolic pressure was overestimated at proximal locations by $1 \%$ (ie, $2 \mathrm{mmHg}$ ) and underestimated by $3 \%$ at distal locations (ie, $4 \mathrm{mmHg}$ ). Boileau et al hypothesized that these differences were caused by the anatomical configuration of the ADAN56 model, which makes the model more sensitive to the coupling strategy at junctions. They also hypothesized that the discrepancies found in the STM model were the result of neglecting the dynamic part of the pressure $\left(\frac{1}{2} \rho u^{2}\right)$ at junctions. ${ }^{9}$ Carson et al ${ }^{16}$ demonstrated that adding the dynamic pressure at junctions to the STM model indeed eliminated the differences found by Boileau et al. ${ }^{9}$

Carson et $\mathrm{al}^{16}$ proposed two methods to constrain the conservation of dynamic pressure at vessel junctions: Lagrange multipliers and a penalty method. Despite the fact that these correction methods showed good results, there are some downsides to these methods. Because the Lagrange multiplier method introduces additional degrees of freedom to the solution vector, the matrix needs to be expanded, which requires a more extensive storage. ${ }^{17}$ For the penalty method, on the other hand, it is difficult to choose the required penalty parameter, the so-called penalty number. ${ }^{16} \mathrm{~A}$ high penalty number is needed to enforce the dynamic pressure constraints; however, if the penalty number is too high, the global matrix becomes ill conditioned with respect to inversion. ${ }^{17}$

The aim of this study is to develop a new element that, on the one hand, is in line with the approach by Kroon et $\mathrm{al}^{15}$ and on the other hand, straightforwardly implements the dynamic part of the pressure in the STM model. The approach is based on the fact that $0 \mathrm{D}$ and 1D elements are cast in the same form. This results in a simplified numerical scheme, in which pressure is the only state variable. After the pressures are calculated on nodal level, the flow can be calculated on element level. This approach allows for flexible coupling between 0D and 1D elements, without the need for additional degrees of freedom or penalty functions. To demonstrate our simplified numerical scheme, we benchmarked our implementations with the ADAN56 geometry presented by Boileau et al. ${ }^{9}$

\section{2 | METHODS}

\section{1 | One-dimensional network model}

In this section, first, we describe the 1D computational domain (ADAN56) that consists of connected 1D arterial elements and is closed with 3-element windkessel elements at truncating parts. At vascular junctions, a new 0D element is introduced (Figure 1). Secondly, we present the assembly of the equations for all different elements. In addition, the numerical method used to solve the scheme is explained. Finally, the simulations performed and their analysis are described.

\subsection{1 | One-dimensional computational domain}

Because Boileau et $\mathrm{al}^{9}$ showed only discrepancies for the STM method in the ADAN56 geometry, we chose to use the ADAN56 model as well. The ADAN56 model contains 56 large vessels of the human arterial system. It originates at the 


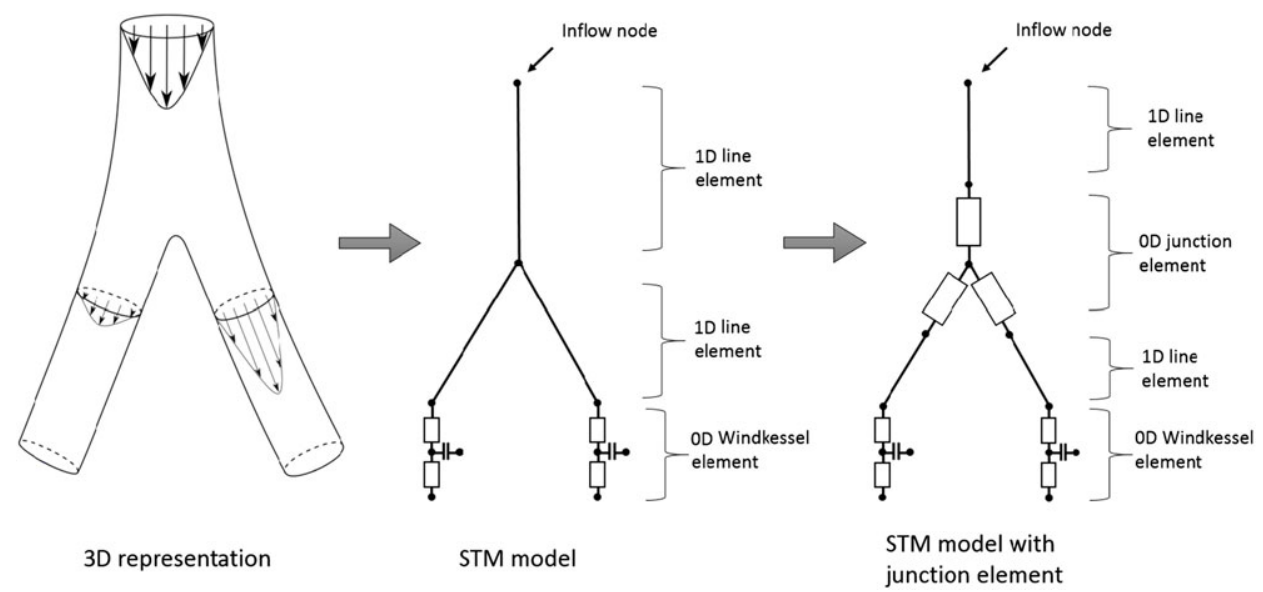

FIGURE 1 The implementation of the 0D junction element in a simple bifurcation geometry

aortic arch and is truncated at the internal carotid, the tibial, and the radial arteries. Furthermore, it is truncated at major organs, such as the kidneys, liver, and lungs. For details on parameters and arterial tree data, the reader is referred to Boileau et al, ${ }^{9}$ which also contains supplementary data containing the results of the 6 numerical schemes.

\subsection{2 | One-dimensional arterial element}

Each arterial element was modeled as a deformable tube, representing a blood vessel whose mechanical properties are described as a function of the axial coordinate $z$. In large arteries, the relation between pressure $p(\mathrm{~Pa})$, flow $q\left(\mathrm{~m}^{3} \mathrm{~s}^{-1}\right)$, and cross-sectional area $\mathrm{A}\left(\mathrm{m}^{2}\right)$ can be modeled using the conservation of mass, the momentum balance equation, and a constitutive law. Here, the area is a function of the pressure, which in turn is a function of the axial location $z$ and time $t$, ie, $A=A(p(z, t))$. We further assumed the vessel wall to be impermeable and the blood to be an incompressible Newtonian fluid. Subsequently, by integrating the mass and momentum equations over the cross-sectional area, we obtained the following $1 \mathrm{D}$ equations ${ }^{6}$ :

$$
\begin{gathered}
\frac{\partial A}{\partial p} \frac{\partial p}{\partial t}+\frac{\partial q}{\partial z}=0 \\
\frac{\partial q}{\partial t}+\left(1+\delta_{S}\right) \frac{\partial}{\partial z}\left(\frac{q^{2}}{A}\right)+\frac{A}{\rho} \frac{\partial p}{\partial z}=\frac{f}{\rho},
\end{gathered}
$$

where $t$ describes time, $\rho$ is the blood density, $\frac{\partial A}{\partial p}$ is the partial derivative of area to pressure, and $f$ is the frictional force per unit length. Following the approach of Boileau et $\mathrm{al},{ }^{9}$ a flat velocity profile was used for the approximation of the advection term, which resulted in a Stokes-layer related thickness of $\delta_{s}=0$. Again, following Boileau et al, ${ }^{9}$ the frictional force per unit length $f$ is given by:

$$
f=-2(\zeta+2) \mu \pi \frac{q}{A},
$$

where $\mu$ is the dynamic viscosity and $\zeta$ is a given constant for a specific velocity profile. For a derivation of (3), the reader is referred to Hughes and Lubliner ${ }^{18}$ and Sherwin et al. ${ }^{19}$ For the friction force, the velocity profile order $\zeta$ was set to 2 by Boileau et al, ${ }^{9}$ which corresponds to a parabolic profile. This mismatch in velocity profiles between the approximation of the advection term and the frictional force can be resolved by using the approximate velocity profile function proposed by Bessems et al, ${ }^{6}$ which also captures the possible phase difference between the viscous boundary layer and the central core. However, the approach by Boileau et $\mathrm{al}^{9}$ was maintained to be able to compare the results.

The relationship between pressure and area, which accounts for the fluid structure interaction of the system, is described as follows ${ }^{9,20}$ :

$$
p=p_{e x t}+p_{d}+\frac{\beta}{A_{d}}\left(\sqrt{A}-\sqrt{A_{d}}\right)
$$




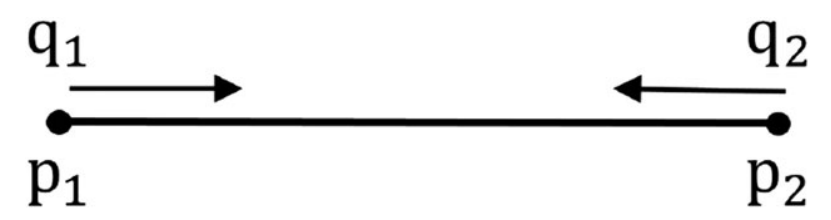

FIGURE 2 Discretization of 1D line element with $p_{i}$ and $q_{i}$ the nodal pressure and flow, respectively

with $p_{\text {ext }}$ the external pressure, $p_{d}$ the diastolic pressure, and $A_{d}$ the diastolic area. In this study, $p_{\text {ext }}$ and $p_{d}$ were set to 0 and $10 \mathrm{kPa}$, respectively. ${ }^{9}$ Here, the parameter $\beta(z)$ accounts for the material properties, which is given by ${ }^{9,20}$ :

$$
\beta(z)=\frac{4}{3} \sqrt{\pi} E h,
$$

with $E$ and $h$ the Young modulus and wall thickness, respectively. Using (4), area vessel compliance can be written as:

$$
\frac{\partial A}{\partial p}=\left(\frac{2 A_{d}\left(p-p_{e x t}-p_{d}\right)}{\beta}+2 \sqrt{A_{d}}\right) \frac{A_{d}}{\beta} .
$$

\section{2 | Numerical implementation}

First, the momentum and mass equation of each element were spatially integrated along the vessel axis using the trapezium rule. Thereafter, a second-order backward difference scheme to step forward in time was applied. Third, both nodal flows were defined inwards (Figure 2). After performing these 3 steps, we arrive at the following 1D line element:

$$
\left[\begin{array}{ll}
K_{11} & K_{12} \\
K_{21} & K_{22}
\end{array}\right]_{e}^{t}\left[\begin{array}{l}
p_{1} \\
p_{2}
\end{array}\right]_{e}^{t+\Delta t}=\left[\begin{array}{c}
q_{1}^{c} \\
-q_{2}^{c}
\end{array}\right]_{e}^{t+\Delta t}+\left[\begin{array}{l}
f_{1} \\
f_{2}
\end{array}\right]_{e}^{t}=\left[\begin{array}{l}
q_{1} \\
q_{2}
\end{array}\right]_{e}^{t+\Delta t}+\left[\begin{array}{l}
f_{1} \\
f_{2}
\end{array}\right]_{e}^{t},
$$

where column vectors $p_{e}$ and $q$ are the nodal point pressures and flows, respectively. Matrix $\mathbf{K}_{e}$ is the element matrix and $f$ is the element right-hand side. Furthermore, $q_{i}^{c}$ denotes the conventional flow definition, and $q_{i}$ denotes the inwards

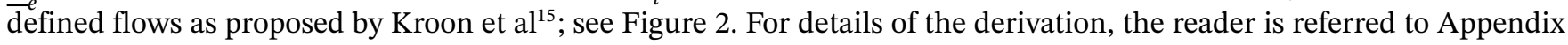
A. Equation 7 can be rewritten in the general form:

$$
\mathbf{K}_{e}^{t, 1 D} \underline{p}_{e}^{t+\Delta t}=\underline{q}_{e}^{t+\Delta t}+\underline{f}_{e}^{t, 1 D} .
$$

\section{3 | Boundary conditions}

For each simulation, inflow was imposed at the root of the network model, ie, the ascending aorta. An inflow signal based on the work of Murgo et $\mathrm{al}^{21}$ was implemented using a truncated Fourier series of the signal. This is the same inflow signal as used by Boileau et $\mathrm{al}^{9}$ and is provided by them in their supplementary material.

At the terminal branches, three-element windkessel elements were modeled. ${ }^{22}$ A windkessel element simulates the effect of the peripheral impedance. It consists of a resistor $R_{1}$ in series with both a resistor $R_{2}$ and a compliance $C$ as shown in Figure 3. The resistance $R_{1}$ is equal to the characteristic impedance of the terminal branch, minimizing wave reflections for high frequencies. ${ }^{22}$ In addition, it holds that $R_{1}+R_{2}=R_{p}$, where $R_{p}$ is the peripheral resistance that is defined by the ratio between the mean arterial pressure and the mean flow through that specific part of the body. Finally, $C$ mimics the peripheral compliance. The windkessel element is given by the following differential equation:

$$
q\left(1+\frac{R_{1}}{R_{2}}\right)+C R_{1} \frac{\partial q}{\partial t}=\frac{p}{R_{2}}+C \frac{\partial p}{\partial t} .
$$

For each subelement of the windkessel element, again, two nodal point pressures and two nodal point flows with inward-directing flow were defined (Figure 3). The extravascular pressure $\left(p_{4}\right)$ and the venous pressure $\left(p_{3}\right)$ were both taken at $0 \mathrm{~Pa}$. Using the same approach as for a $1 \mathrm{D}$ line element, the windkessel element can be cast in the same form as in (8) (see Appendix B):

$$
\mathbf{K}_{e}^{t, 0 D} \underline{p}_{e}^{t+\Delta t}=\underline{f}_{e}^{t, 0 D}+\underline{q}_{e}^{t+\Delta t}
$$




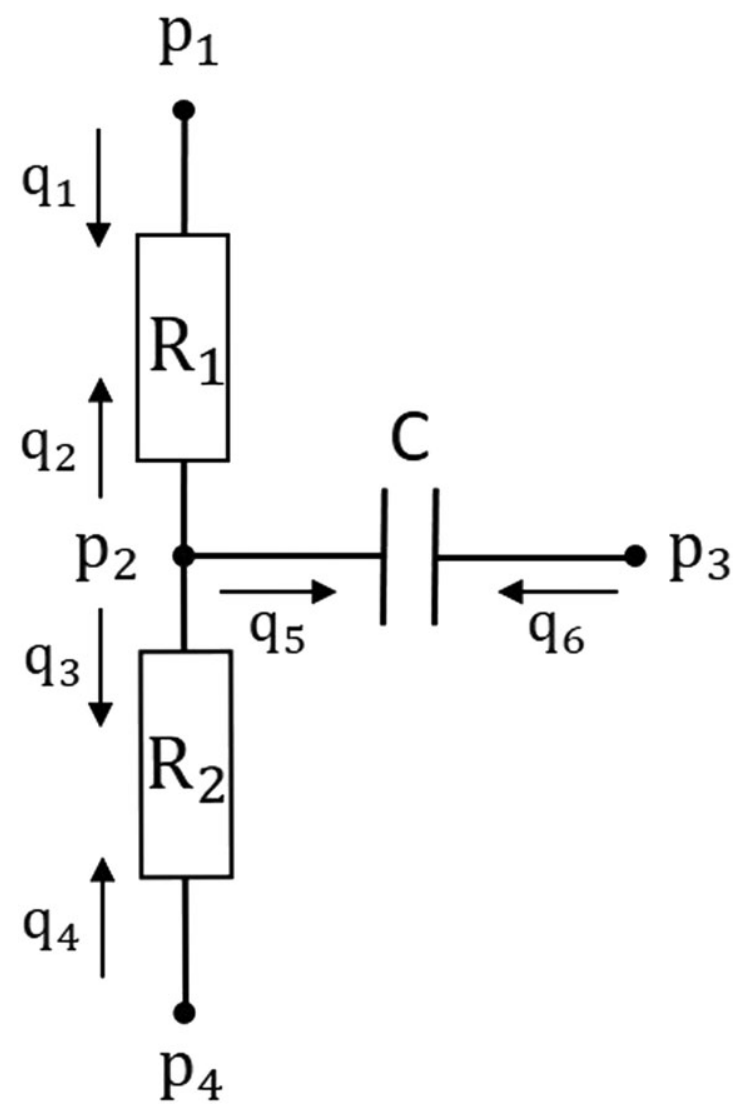

FIGURE 3 Discretization of OD windkessel with $p_{i}$ and $q_{i}$ the nodal pressure and flow, respectively. $R_{1}$ is the characteristic impedance, $R_{2}$ the peripheral resistance, and $C$ the peripheral compliance

\section{4 | Vessel junctions}

At vessel junctions, three 1D arterial elements need to be connected. Since the element flows are defined inwards in the original STM model presented by Kroon et al, ${ }^{15}$ the assumption that the $\sum q_{i}=0$ is automatically satisfied. Consequently, the nodal pressures are coupled directly without the need for additional coupling equations, resulting in a shared node at junctions, as can be seen in Figure 1. This continuity of static pressure between the three branches is expressed by:

$$
p_{1}=p_{2}=p_{3} .
$$

However, to be able to compare the STM model with the other five numerical schemes, continuity of total pressure is required at vessel junctions. Therefore, the dynamic part of the pressure should be added to each term in (11), yielding:

$$
p_{1}+\frac{1}{2} \rho u_{1}^{2}=p_{2}+\frac{1}{2} \rho u_{2}^{2}=p_{3}+\frac{1}{2} \rho u_{3}^{2},
$$

where $u$ is the mean blood velocity, $\rho$ the blood density, and $p$ and $\frac{1}{2} \rho u^{2}$ are the static and dynamic components of pressure, respectively. Here, $\hat{p}=p+\frac{1}{2} \rho u^{2}$ is called the total pressure. The continuity of total pressure can be described by a pressure loss or gain, which results from the difference in mean velocity between branches at junctions:

$$
\begin{aligned}
& p_{1}-p_{2}=\frac{1}{2} \rho\left(u_{2}^{2}-u_{1}^{2}\right), \\
& p_{1}-p_{3}=\frac{1}{2} \rho\left(u_{3}^{2}-u_{1}^{2}\right), \\
& p_{2}-p_{3}=\frac{1}{2} \rho\left(u_{3}^{2}-u_{2}^{2}\right) .
\end{aligned}
$$

To implement these additional pressure differences (losses or gains), a 0D element was introduced and implemented at junctions as shown in Figure 1. This 0D junction element is presented in more detail in Figure 4. To define the 0D junction 


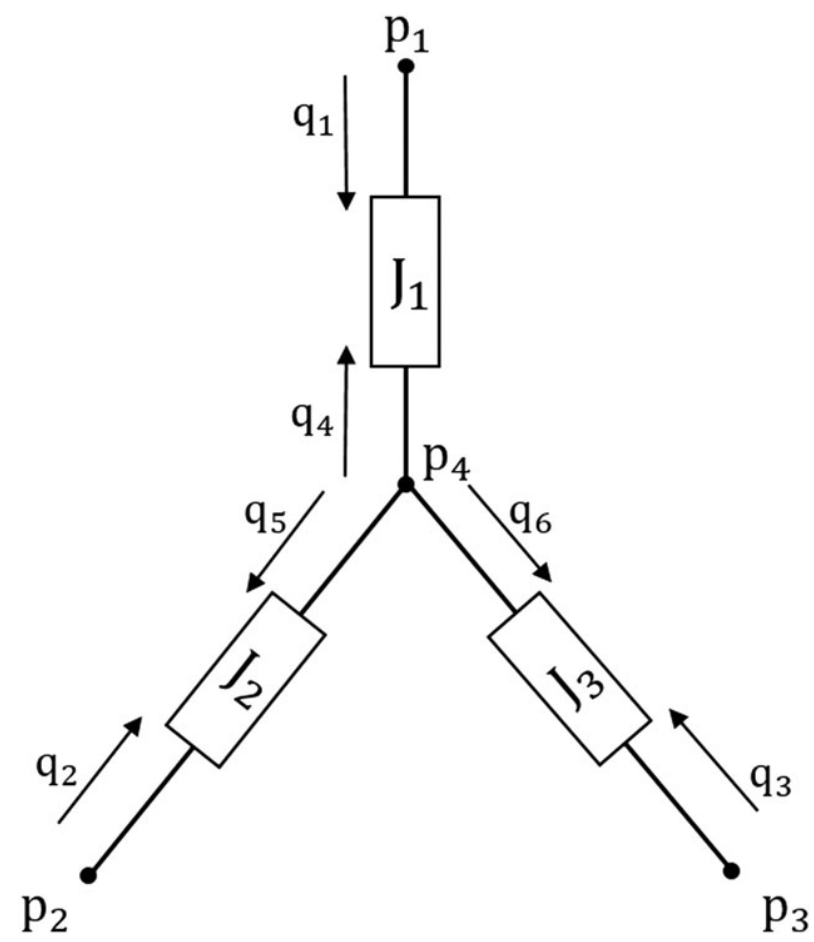

FIGURE 4 Discretization of OD junction element with pi and qi the nodal pressure and flow, respectively. J are the junction components

element, we denoted one branch as the reference vessel. In this study, the reference vessel carries the total flow entering the element (in case of a bifurcating flow) or leaving the element (in case of a merging flow). However, an arbitrary branch can be chosen. We then assumed that there are no additional pressure differences over the reference vessel, leading to the pressure in the reference vessel being approximately equal to the pressure at the central node $p_{4}$. To prevent an extra pressure drop from occurring, this component was modeled as a small resistance with $J=1 \mathrm{kgm}^{-4} \mathrm{~s}^{-1}$. The value of $J=1 \mathrm{kgm}^{-4} \mathrm{~s}^{-1}$ was chosen such that this value is much smaller than the Poiseuille resistance of the reference vessel. For the other two branches, the pressure differences caused by the addition of the dynamic pressure were defined with respect to the reference vessel:

$$
p_{i}-p_{4}=\frac{1}{2} \rho\left(\frac{q_{r}^{2}}{A_{r}^{2}}-\frac{q_{i}^{2}}{A_{i}^{2}}\right)
$$

with $q_{i}$ and $A_{i}$ the flow rate and area of the $i$ th branch, respectively. Now, each component $J_{i}$ of this junction element relates the pressure of the central node $p_{4}$ to the external pressure nodes $p_{1}, p_{2}$, and $p_{3}$ of the three branches.

After linearization of (14) using the Newton-Raphson method, we obtained (see Appendix C):

$$
p_{i}^{t+\Delta t}-p_{4}^{t+\Delta t}=-\rho \frac{q_{i}^{t}}{\left(A_{i}^{t}\right)^{2}} q_{i}^{t+\Delta t}+\frac{\rho}{2} \frac{\left(q_{i}^{t}\right)^{2}}{\left(A_{i}^{t}\right)^{2}}+\frac{\rho}{2} \frac{\left(q_{r}^{t}\right)^{2}}{\left(A_{r}^{t}\right)^{2}}, \mathrm{i}=1,2,3,
$$

where superscript $t$ is the approximation of the previous time step and $t+\Delta t$ the approximation of the current time step. This can be summarized to the general form:

$$
p_{i}^{t+\Delta t}-p_{4}^{t+\Delta t}=J_{i}^{t} q_{i}^{t+\Delta t}+g_{i}^{t} \quad \mathrm{i}=1,2,3,
$$

with $g_{i}$ the last 2 terms of the right-hand side of (15). We then divided (16) by $J_{i}$ resulting in:

$$
\frac{1}{J_{i}}\left(p_{i}^{t+\Delta t}-p_{4}^{t+\Delta t}\right)=q_{i}^{t+\Delta t}+f_{i}^{t}, \quad \mathrm{i}=1,2,3,
$$


with $f_{i}^{t}=\frac{g_{i}^{t}}{J_{i}}$. Now, component $J_{1}$ in Figure 4 could be cast into the form:

$$
\left[\begin{array}{cc}
\frac{1}{J_{1}} & -\frac{1}{J_{1}} \\
-\frac{1}{J_{1}} & \frac{1}{J_{1}}
\end{array}\right]_{e}^{t}\left[\begin{array}{l}
p_{1} \\
p_{4}
\end{array}\right]_{e}^{t+\Delta t}=\left[\begin{array}{c}
q_{1}^{c} \\
-q_{4}^{c}
\end{array}\right]_{e}^{t+\Delta t}+\left[\begin{array}{c}
f_{1} \\
-f_{1}
\end{array}\right]_{e}^{t}=\left[\begin{array}{l}
q_{1} \\
q_{4}
\end{array}\right]_{e}^{t+\Delta t}+\left[\begin{array}{c}
f_{1} \\
-f_{1}
\end{array}\right]_{e}^{t},
$$

where $q_{i}^{c}$ once again denotes the conventional notation and $q_{i}$ the notation as presented by Kroon et al. ${ }^{15}$

Similarly, component $J_{2}$ and $J_{3}$ were cast into the same form. By assembling the 3 junction components, the total element matrix is:

$$
\left[\begin{array}{cccc}
\frac{1}{J_{1}} & 0 & 0 & -\frac{1}{J_{1}} \\
0 & \frac{1}{J_{2}} & 0 & -\frac{1}{J_{2}} \\
0 & 0 & \frac{1}{J_{3}} & -\frac{1}{J_{3}} \\
-\frac{1}{J_{1}} & -\frac{1}{J_{2}} & -\frac{1}{J_{3}} & \frac{1}{J_{1}}+\frac{1}{J_{2}}+\frac{1}{J_{3}}
\end{array}\right]_{e}^{t}\left[\begin{array}{c}
p_{1} \\
p_{2} \\
p_{3} \\
p_{4}
\end{array}\right]_{e}^{t+\Delta t}=\left[\begin{array}{c}
q_{1} \\
q_{2} \\
q_{3} \\
q_{4}+q_{5}+q_{6}
\end{array}\right]_{e}^{t+\Delta t}+\left[\begin{array}{c}
f_{1} \\
f_{2} \\
f_{3} \\
-f_{1}-f_{2}-f_{3}
\end{array}\right]_{e}^{t},
$$

with $q_{4}+q_{5}+q_{6}=0$ due to conservation of mass. Finally, the 0D junction element (defined by (19)) was cast into the same form as the $1 \mathrm{D}$ line elements (defined by (8)) and the 0D windkessel element (as given by (10)):

$$
\mathbf{K}_{e}^{t, 0 D} \underline{p}_{e}^{t+\Delta t}=\underline{f}_{e}^{t, 0 D}+\underline{q}_{e}^{t+\Delta t}
$$

\section{5 | Assembling all elements and numerical method}

Noticing that the element matrices (8), (10), and (20) were all cast in the same form, assembly of the large system of equations involved summation of all 1D element equations and $0 \mathrm{D}$ element equations. The assembly process is here demonstrated for 2 line elements in series. Consider element 1 with pressure nodes $p_{1}$ and $p_{2}$ and flows $q_{1}$ and $q_{2}$, and element 2 with pressure nodes $p_{2}$ and $p_{3}$ and flows $q_{3}$ and $q_{4}$. The 2 element matrices of the 2 line elements defined by (7) can be assembled to the following system:

$$
\left[\begin{array}{ccc}
K_{11}^{1} & K_{12}^{1} & 0 \\
K_{21}^{1} & K_{22}^{1}+K_{11}^{2} & K_{12}^{2} \\
0 & K_{21}^{2} & K_{22}^{2}
\end{array}\right]_{e}^{t}\left[\begin{array}{c}
p_{1} \\
p_{2} \\
p_{3}
\end{array}\right]_{e}^{t+\Delta t}=\left[\begin{array}{c}
q_{1} \\
q_{2}+q_{3} \\
q_{4}
\end{array}\right]_{e}^{t+\Delta t}+\left[\begin{array}{c}
f_{1}^{1} \\
f_{2}^{1}+f_{1}^{2} \\
f_{2}^{2}
\end{array}\right]_{e}^{t}
$$

where the superscripts of $K_{i}$ and $f_{i}$ represent the elements they belong to. Conservation of mass requires the outflow of element $1\left(q_{2}\right)$ to be equal to the inflow of element $2\left(q_{3}\right)$, resulting in $q_{2}+q_{3}=0$. Therefore, the internal node in this example has value of 0 and only the flows $q_{1}$ and $q_{4}$ (boundary conditions) are unknown. At the inlet of the arterial tree, the nodal flow was prescribed, and for terminal nodes, a constant pressure was prescribed (essential boundary condition), resulting in only one degree of freedom for every node. The final assembled matrix containing the 1D line, 0D windkessel, and $\mathrm{OD}$ junction elements is given by:

$$
\mathbf{K}^{t} \underline{p}^{t+\Delta t}=\underline{f}^{t}+\underline{q}^{e x, t+\Delta t}
$$

The system was solved using the second-order backward difference scheme for time discretization, ${ }^{15}$ with a timestep of $0.5 \mathrm{~ms}$ and element size of $0.5 \mathrm{~cm}$. The finite element package SEPRAN (version 0610) was used to solve the system, while Matlab R2016b (The MathWorks, Inc, Natick, Massachusetts) was used for preprocessing and postprocessing. After 
calculation of pressures and flows, the simulation proceeded to the next time step. The process was repeated until cardiac cycle time was reached. At this point, the nodal point maximum relative root-mean-squared norm of both pressure and flow, denoted $\varepsilon_{p}$ and $\varepsilon_{q}$, respectively, were calculated compared with the previous cardiac cycle. Each simulation was run for 10 cycles, resulting in a convergence norm $\varepsilon<10^{-3}$.

\section{6 | Simulation and analysis}

The following simulations were run for the ADAN56 benchmark case: (1) The original STM method (STM), (2) STM + new 0D junction element (STM-J), and (3) the FEM scheme presented in Boileau et al. ${ }^{9}$ The other four schemes shown in Boileau et $\mathrm{al}^{9}$ that already have continuity of total pressure at vessel junctions gave consistent results with the FEM method and are therefore not shown. The results of the five other benchmark cases are not shown as the STM scheme already showed good agreement compared with the other methods, even without the addition of the dynamic part of the pressure, and because these cases were hardly effected by the addition of the new 0D junction element, as expected.

First, flow rate and pressure waveforms are shown for the aortic arch I, the right internal carotid, the right renal, and the right anterior tibial. These locations were chosen to show the effect of adding the dynamic pressure when moving more distal from the heart, both upwards and downwards. We then calculate a variety of "errors" that are calculated to compare the results using the FEM as the golden standard: $\mathcal{E}_{p}^{R M S}$ and $\mathcal{E}_{q}^{R M S}$ are the root mean square relative error for pressure and flow, $\mathcal{E}_{p}^{M A X}$ and $\mathcal{E}_{q}^{M A X}$ are the maximum relative errors in pressure and flow, $\mathcal{E}_{p}^{S Y S}$ and $\mathcal{E}_{q}^{S Y S}$ are the errors in systolic pressure and flow, and $\mathcal{E}_{p}^{\text {DIAS }}$ and $\mathcal{E}_{q}^{\text {DIAS }}$ are the errors in diastolic pressure and flow, respectively. These are calculated with the following equations:

$$
\begin{gathered}
\mathcal{E}_{p}^{R M S}=\sqrt{\frac{1}{n} \sum_{i=1}^{n}\left(\frac{p_{i}^{S T M}-p_{i}^{F E M}}{p_{i}^{F E M}}\right)^{2}}, \quad \mathcal{E}_{q}^{R M S}=\sqrt{\frac{1}{n} \sum_{i=1}^{n}\left(\frac{q_{i}^{S T M}-q_{i}^{F E M}}{\max \left(q_{i}^{F E M}\right)}\right)^{2},} \\
\mathcal{E}_{p}^{M A X}=\max _{i}\left|\frac{p_{i}^{S T M}-p_{i}^{F E M}}{p_{i}^{F E M}}\right|, \quad \mathcal{E}_{q}^{M A X}=\max _{i}\left|\frac{q_{i}^{S T M}-q_{i}^{F E M}}{\max \left(q_{i}^{F E M}\right)}\right|, \\
\mathcal{E}_{p}^{S Y S}=\frac{\max \left(p^{S T M}\right)-\max \left(p^{F E M}\right)}{\max \left(p^{F E M}\right)}, \quad \mathcal{E}_{q}^{S Y S}=\frac{\max \left(q^{S T M}\right)-\max \left(q^{F E M}\right)}{\max \left(q^{F E M}\right)}, \\
\mathcal{E}_{p}^{D I A S}=\frac{\min \left(p^{S T M}\right)-\min \left(p^{F E M}\right)}{\min \left(p^{F E M}\right)}, \quad \mathcal{E}_{q}^{D I A S}=\frac{\min \left(q^{S T M}\right)-\min \left(q^{F E M}\right)}{\min \left(q^{F E M}\right)},
\end{gathered}
$$

with value $n$ the maximum number of time steps within one cardiac cycle. Finally, we show the propagation of the systolic, diastolic, and mean pressure along the aorta, because this captures the general physiological features of pulse wave propagation.

\section{3 | RESULTS}

Figure 5 shows the flow rate and pressure waveforms of the four different positions in the ADAN56 network. Though in each panel of Figure 5 the waveforms as derived by STM, STM-J, and FEM are plotted, the latter two signals can often not be discriminated, indicating their good agreement. This good agreement can also be seen when looking at the errors in Table 1. In the original STM model, the root mean square relative errors $\mathcal{E}^{R M S}$ ranged from $0.51 \%$ to $4.10 \%$, while with the extended STM-J model, the root mean square relative errors $\mathcal{E}^{R M S}$ have a fourfold reduction and range from $0.12 \%$ to $0.67 \%$. Furthermore, the systolic and diastolic pressure are in better agreement for the STM-J model, with a fourfold error 

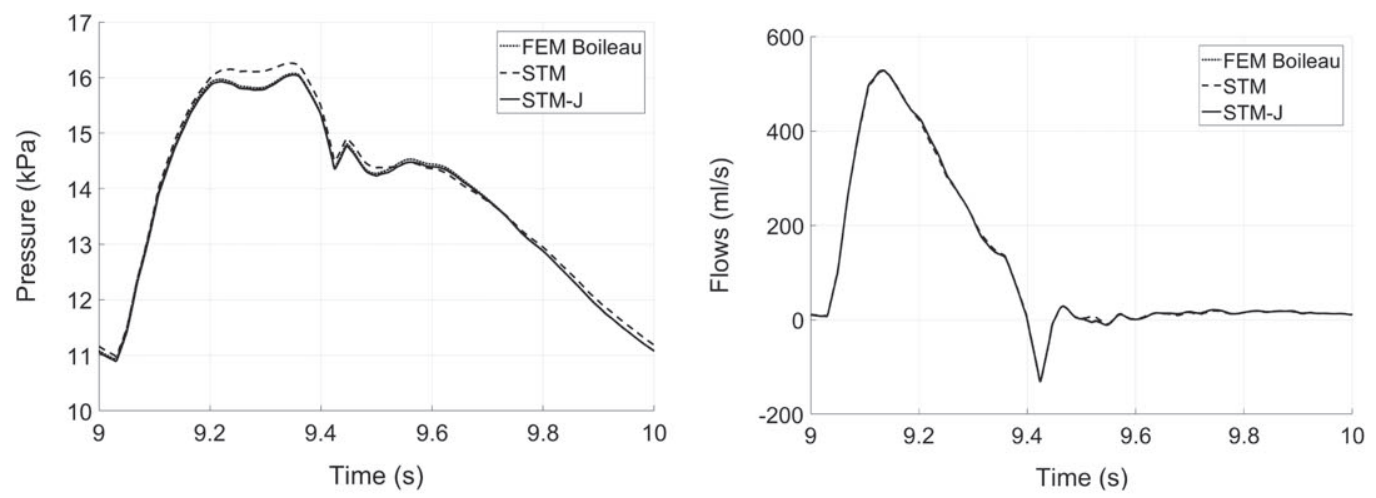

Pressure in Aortic Arch I
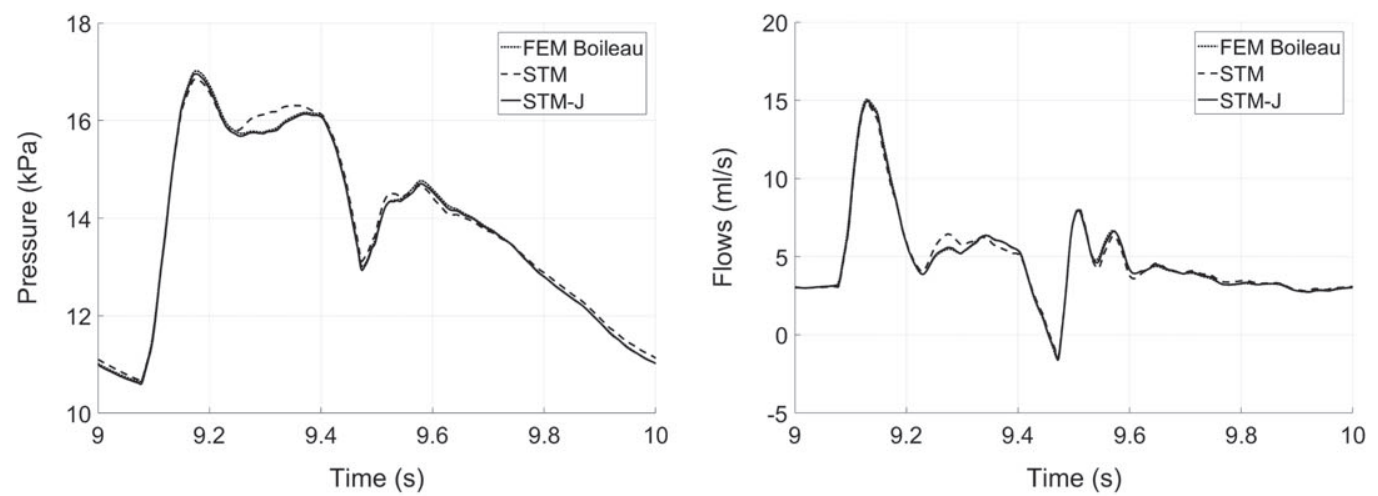

Pressure in Right Internal Carotid

Flow Rate in Right Internal Carotid
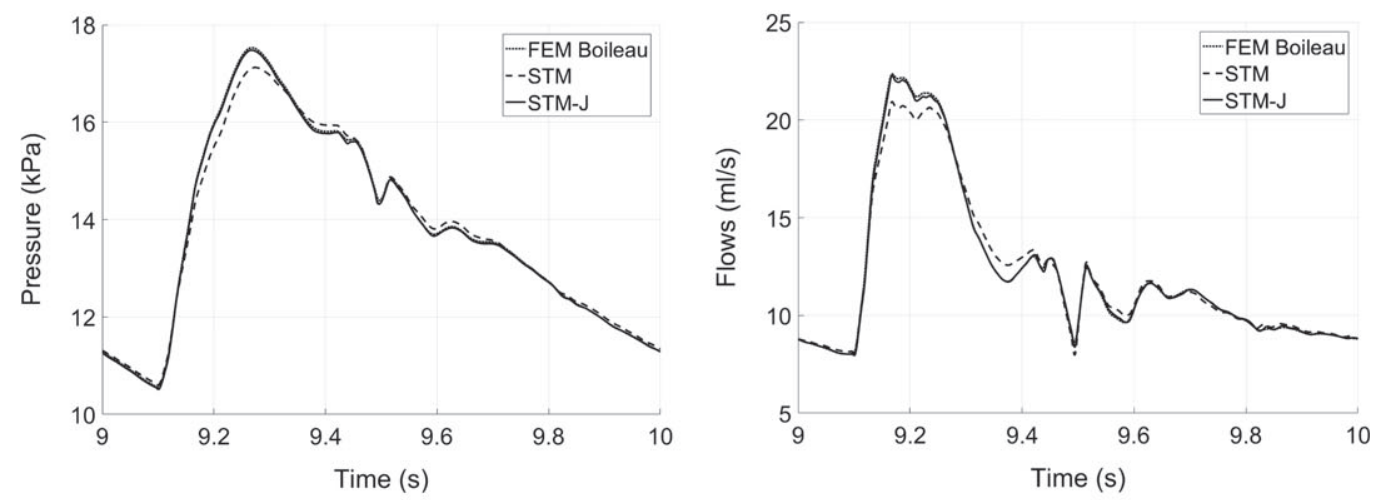

Pressure in Right Renal

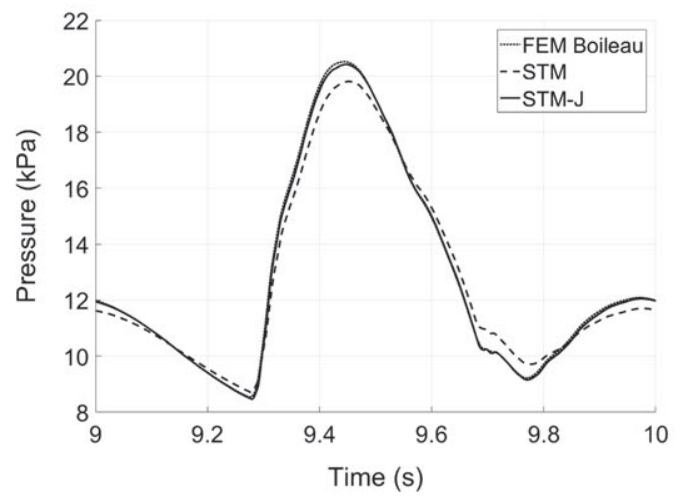

Pressure in Right Anterior Tibial

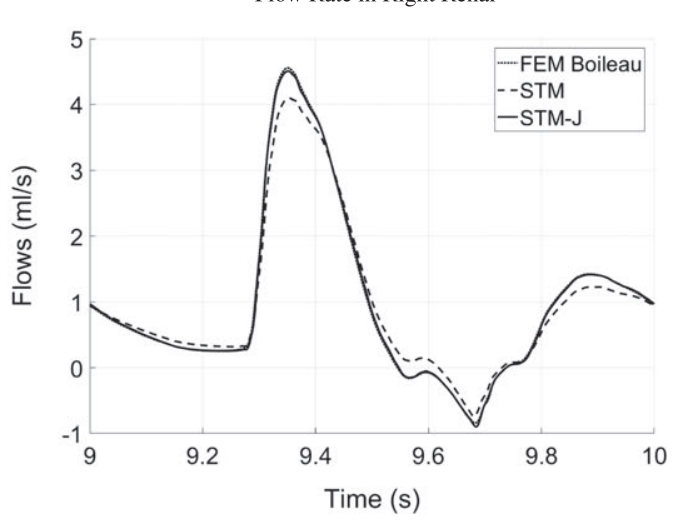

Flow Rate in Right Anterior Tibial

FIGURE 5 Pressure and flow rate waveforms, comparing the finite element method (FEM), simplified trapezium rule method (STM), and STM with junction (STM-J) 
TABLE 1 Relative error with respect to the finite element method at the midpoint of the vessels in percent

\begin{tabular}{|c|c|c|c|c|c|c|c|c|c|}
\hline Arterial Segment & Method & $\mathcal{E}_{p}^{R M S}, \%$ & $\mathcal{E}_{p}^{M A X}, \%$ & $\mathcal{E}_{p}^{S Y S}, \%$ & $\mathcal{E}_{p}^{D I A S}, \%$ & $\mathcal{E}_{q}^{R M S}, \%$ & $\mathcal{E}_{q}^{M A X}, \%$ & $\mathcal{E}_{q}^{S Y S}, \%$ & $\mathcal{E}_{q}^{D I A S}, \%$ \\
\hline \multirow[t]{2}{*}{ Aortic arch I } & STM & 0.83 & 1.87 & 1.14 & 0.50 & 0.51 & 1.94 & -0.17 & 0.34 \\
\hline & STM-J & 0.25 & 0.55 & -0.16 & -0.34 & 0.12 & 0.41 & 0.13 & 1.38 \\
\hline \multirow[t]{2}{*}{ Right internal carotid } & STM & 0.89 & 2.35 & -1.00 & 0.13 & 1.98 & 6.41 & -1.51 & -3.11 \\
\hline & STM-J & 0.27 & 0.69 & -0.38 & -0.49 & 0.56 & 2.23 & 0.82 & 6.90 \\
\hline \multirow[t]{2}{*}{ Right renal } & STM & 1.11 & 3.09 & -2.31 & 0.39 & 2.25 & 6.90 & -6.35 & -0.47 \\
\hline & STM-J & 0.24 & 0.63 & -0.29 & -0.44 & 0.41 & 1.57 & -0.33 & -0.74 \\
\hline \multirow[t]{2}{*}{ Right anterior tibial } & STM & 3.39 & 6.95 & -3.44 & -1.90 & 4.10 & 12.54 & -10.02 & -11.13 \\
\hline & STM-J & 0.67 & 2.99 & -0.47 & -0.94 & 0.64 & 2.68 & -1.04 & -7.16 \\
\hline
\end{tabular}

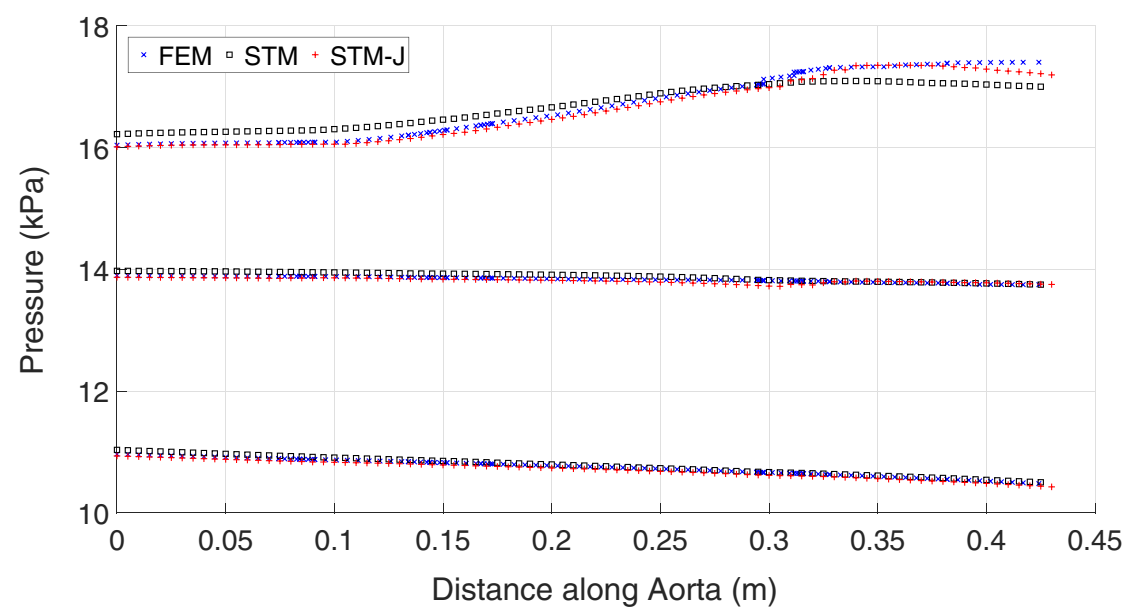

FIGURE 6 Systolic, mean, and diastolic pressure along the aorta for the finite element method (FEM), the simplified trapezium method (STM), and the STM with new junction element (STM-J)

reduction in the aortic arch I. This error reduction increases for more distal vessels, as seen for the right anterior tibial, where the relative systolic error decreases tenfold.

Figure 6 shows the propagation of the systolic, mean, and diastolic pressure along the aorta. While the STM shows an overestimation of $1 \%$ of the systolic pressure in the beginning of the aorta and an underestimation of 3\% when moving more distally, the new STM-J model resolves this error for the systolic pressure. This can be seen by a relative error that is lower than $0.5 \%$ when comparing the systolic pressure of the STM-J model along the aorta with the systolic pressure of the FEM model.

\section{4 | DISCUSSION}

The aim of this study was to introduce a new 0D junction element to incorporate the dynamic part of the pressure in the already existing STM model proposed by Kroon et al. ${ }^{15}$ Using the new coupling strategy described in Section 2, the total pressure $\left(p+\frac{1}{2} \rho u^{2}\right)$ was constrained at vessel junctions without the need of expanding the global matrix with Lagrange multipliers or introducing a penalty function as previously published by Carson et al. ${ }^{16}$ After implementing the new 0D junction element, the pressure and flow waveforms showed good agreement with the results of the five other numerical schemes described by Boileau et al, ${ }^{9}$ (see Figure 5) that already had incorporated dynamic pressure at vessel junctions.

By following the inward-directed flow approach as described by Kroon et $a l,{ }^{15}$ we showed the flexibility of the STM model and the relative ease of implementing a new element. By casting every element in the same form as in (22) and defining flow inwards at element level, no extra coupling equations were needed between elements and computational speed and efficiency were maintained. This approach was recently also used by Quereshi et al, ${ }^{23}$ who modeled the pulmonary and venous circulation. Moreover, this approach can also easily be used to implement other vascular concepts with nonlinear pressure-flow rate relations, such as a stenosis, a heart pump, or the calf muscle pump function. ${ }^{2}$ Although we demonstrated the ease of adding the dynamic part of the pressure to the STM method, one might argue the importance of adding 
the dynamic part. The influence of pressure losses at junctions becomes of more importance when the magnitude of the blood flow velocity is increased. Mynard et al ${ }^{24}$ estimated a pressure loss of $1 \mathrm{mmHg}$ (approximately 0.5kPa) after 3 typical diverging or converging junctions under resting circumstances with a maximum velocity of $0.5 \mathrm{~m} / \mathrm{s}$. During exercise, with a maximum aortic blood flow velocity of $2.0 \mathrm{~m} / \mathrm{s}$, they estimated a pressure loss of $17 \mathrm{mmHg}$ (approximately $2.3 \mathrm{kPa}$ ) after 3 junctions. This dependence on blood flow velocity and number of junctions is even larger in the pulmonary arterial and the venous network, which consists of many generations of junctions. ${ }^{23}$

Mynard et $\mathrm{al}^{24}$ showed that both methods of coupling (with or without the dynamic part) did not result in a reliable estimation of the pressure at junctions compared with 3D simulations and that neither method could be judged as generally superior to the other. While conservation of dynamic pressure will affect the pressure losses at vascular junctions, an extra energy dissipation that depends on the junction geometry and flow conditions is also present. This dissipation may counterbalance the pressure losses from the addition of the dynamic part of the pressure. The extra pressure loss $K_{1,2}\left(\frac{1}{2} \rho u_{c o m}^{2}\right)$ is caused by a change of direction of flow and will result in an extra pressure loss due to the formation of vortices at the entrance region of a bifurcation. ${ }^{23}$ Therefore, (13) should be written as:

$$
p_{1}-p_{2}=\frac{1}{2} \rho\left(u_{2}^{2}-u_{1}^{2}\right)+K_{1,2}\left(\frac{1}{2} \rho u_{c o m}^{2}\right),
$$

with $u_{\text {com }}$ the velocity in the common branch and $K_{1,2}$ a constant that is dependent on the junction geometry and flow conditions. Mynard et $\mathrm{al}^{24}$ proposed a method for estimating the constant $K_{1,2}$ and thus the calculation of the extra pressure losses at junctions. Although not modeled in the current $0 \mathrm{D}$ junction element, this extra pressure loss can easily be added to the current element following the approach proposed in this article.

Though the current $0 \mathrm{D}$ junction element only connects three line elements, it can easily be expanded to junctions with more than three branches. However, because these junctions with more than three branches are mostly found in smaller vessels, the assumptions that are made for 1D modeling of the larger blood vessels, eg, Newtonian fluid, possibly no longer hold. ${ }^{6}$ Extending the junction element to more branches therefore has to be done with care.

Choosing the right coupling strategy is shown to be important on the wave propagation. The possible addition of dynamic part of the pressure $\left(\frac{1}{2} \rho u^{2}\right)$ and the extra pressure loss $\left(K_{1,2}\left(\frac{1}{2} \rho u_{c o m}^{2}\right)\right)$ must be evaluated for every application. While for simple bifurcations, the differences between coupling strategies are negligible, these differences become larger for arterial networks with an increasing number of generations and with increasing blood velocity. ${ }^{9,24}$ However, the influence of the pressure coupling can be much smaller compared with changes by choosing a different velocity profile, a different boundary inflow, a different constitutive law, or the implementation of viscoelastic wall behavior. ${ }^{25}$ Because Newtonian fluid is assumed in most 1D schemes (including ours), blood rheology effects including blood shear-thinning are neglected. ${ }^{26}$ Since we were only interested in implementing a dynamic pressure element and comparing this with already existing models, the lack of such a rheology model is not of importance for our study. Moreover, the geometry used in our study consists of vessels of a large caliber, where blood shear thinning is not as important, because of high shear regions. ${ }^{26}$ Although it is possible to make the model more complex or less complex, ${ }^{25-27}$ it is very important to evaluate model choices, such as the inclusion of rheology or viscoelastic wall behavior, depending on the models application.

\section{5 | CONCLUSION}

The implementation of a $0 \mathrm{D}$ junction element in the simplified trapezium rule method enabled incorporation of the dynamic part of the pressure at vessel junctions. Using the approach by Kroon et al ${ }^{15}$ flexible coupling between 0D and 1D elements is possible and nonlinear pressure-flow equations can be easily incorporated. The extended STM model shows good agreement with the 5 other schemes shown by Boileau et al, ${ }^{9}$ while maintaining computational speed and efficiency.

\section{ORCID}

Tim van den Boom (iD) http://orcid.org/0000-0002-9161-4272

\section{REFERENCES}

1. Ferranti F, Tamburelli V, Antonini G. Rational macromodeling of 1D blood flow in the human cardiovascular system. Int J Numer Meth Biomed Eng. 2015;31:1-17. 
2. Keijsers JMT, Leguy CAD, Huberts W, Narracott AJ, Rittweger J. A 1D pulse wave propagation model of the hemodynamics of calf muscle pump function. Int J Numer Meth Biomed Eng. 2015;31(7):1-20.

3. Qing P, Ruofan W, Bettina R, Guolong C, Jing Y, Axel P, Gangmin N. A one-dimensional mathematical model for studying the pulsatile flow in microvascular networks. J Biomech Eng. 2013;136(1):011009.

4. Huberts W, Bode AS, Kroon W, Planken RN, Tordoir JH, van de Vosse FN, Bosboom EM. A pulse wave propagation model to support decision-making in vascular access planning in the clinic. Med Eng Phys. 2011;34(2):233-248.

5. Marsden A. Simulation based planning of surgical interventions in pediatric cardiology. Phys Fluids. 2013;25:1-20.

6. Bessems D, Rutten M, van de Vosse FN. A wave propagation model of blood flow in large vessels using an approximate velocity profile function. J Fluid Mech. 2007;580:145-168.

7. Perdikaris P, Grinberg L, Karniadakis G. An effective fractal-tree closure model for simulating blood flow in large arterial networks. Ann Biomed Eng. 2014;43:1432-1442.

8. van de Vosse FN, Stergiopulos N. Pulse wave propagation in the arterial tree. Ann Rev Fluid Mech. 2011;43(1):467-499.

9. Boileau E, Nithiarasu P, Blanco JP, et al. A benchmark study for numerical schemes for one dimensional arterial blood flow modelling. Int J Numer Meth Biomed Eng. 2015;31(10):e02732.

10. Thomas CG, Nithiarasu P, Bevan RLT. The locally conservative Galerkin (LCG) method for solving the incompressible Navier Stokes equations. Int J Numer Meth Fluids. 2008;57:1771-1792.

11. Alastruey J, Parker KH, Sherwin SJ. Arterial Pulse Wave Haemodynamics. Lisbon: Virtual PiE Led t/a BHR Group; 2012.

12. Watanabe S, Blanco P, Raúl F. Mathematical model of blood flow in an anatomically detailed arterial network of the arm. ESAIM: Math Modell Numer Anal. 2013;47:961-985.

13. Müller LO, Parés C, Eleuterio FT. Well-balanced high-order numerical schemes for one-dimensional blood flow in vessels with varying mechanical properties. J Comput Phys. 2013;242:53-85.

14. Anderson JD. Computational Fluid Dynamics: The Basics with Applications, McGraw-Hill international editions. Washington DC: McGraw-Hill; 1995.

15. Kroon W, Huberts W, Bosboom M, Van De Vosse FN. A numerical method of reduced complexity for simulating vascular hemodynamics using coupled 0D lumped and 1D wave propagation models. Comput Math Methods Med. 2012;2012:0-10.

16. Carson J, van Loon R. An implicit solver for 1D arterial network models. Int J Numer Meth Biomed Eng. 2016;02837:1-16.

17. Felipa CA. Introduction to Finite Element Methods: Chapter 9 Multifreedom Constraints II; 2004.

18. Hughes TJR, Lubliner J. On the one-dimensional theory of blood flow in the larger vessels. Math Biosci. 1973;18(1-2):161-170.

19. Sherwin SJ, Franke V, Peiró J, Parker K. One-dimensional modelling of a vascular network in space-time variables. $J$ Eng Math. 2003;47(3-4):217-250.

20. Formaggia L, Lamponi D, Quarteroni A. One-dimensional models for blood flow in arteries. J Eng Math. 2003;47(3):251-276.

21. Murgo JP, Westerhof N, Giolma JP, Altobelli SA. Aortic input impedance in normal man: relationship to pressure wave forms. Circulation. 1980;62:105-116.

22. Westerhof N, Elzinga G, Sipkema P. An artificial arterial system for pumping hearts. J Appl Physiol. 1971;31(5):776-81.

23. Qureshi MU, Vaughan GDA, Sainsbury C, Johnson M, Peskin CS, Olufsen MS. Numerical simulation of blood flow and pressure drop in the pulmonary arterial and venous circulation. Biomech Model Mechanobiol. 2014;13(5):1137-1154.

24. Mynard JP, Valen-sendstad K. A unified method for estimating pressure losses at vascular junctions. Int J Numer Meth Biomed Eng. 2015;31(7):1-23.

25. Bessems D, Giannopapa CG, Rutten MCM, van de Vosse FN. Experimental validation of a time-domain-based wave propagation model of blood flow in viscoelastic vessels. J Biomech. 2008;41(2):284-291.

26. Ghigo AR, Lagrée PY, Fullana JM. A time-dependent non-Newtonian extension of a 1D blood flow model. J Nonnewton Fluid Mech. 2018;253:36-49.

27. Reymond P, Merenda F, Perren F, Rufenacht D, Stergiopulos N. Validation of a one-dimensional model of the systemic arterial tree. Am J Physiol Heart Circ Physiol. 2009;297:H208-22.

How to cite this article: van den Boom T, Stevens RRF, Delhaas T, van de Vosse FN, Huberts W. Zero-dimensional lumped approach to incorporate the dynamic part of the pressure at vessel junctions in a 1D wave propagation model. Int J Numer Meth Biomed Engng. 2018;e3116. https://doi.org/10.1002/cnm.3116

\section{APPENDIX A : ONE-DIMENSIONAL LINE ELEMENT EQUATIONS}

First of all, recall the momentum equation of (2):

$$
\frac{\partial q}{\partial t}+\left(1+\delta_{s}\right) \frac{\partial}{\partial z}\left(\frac{q^{2}}{A}\right)+\frac{A}{\rho} \frac{\partial p}{\partial z}=\frac{f}{\rho},
$$


with:

$$
f=-2(\zeta+2) \mu \pi \frac{q}{A}
$$

Also, recall the mass equation:

$$
C_{A} \frac{\partial p}{\partial t}+\frac{\partial q}{\partial z}=0
$$

with $C_{A}=\frac{\partial A}{\partial p}$.

If we set $\zeta=2$ and $\delta_{s}=0$ in (A1), corresponding to Boileau et al, ${ }^{9}$ these equations reduce to:

$$
\frac{\rho}{A} \frac{\partial q}{\partial t}+\frac{\partial p}{\partial z}=-\frac{8 \mu \pi q}{A^{2}}-\frac{\rho}{A} \frac{\partial}{\partial z}\left(\frac{q^{2}}{A}\right)
$$

To discretize and linearize this 1D momentum equation (A4) and the conservation of mass equation (1), the vessels are first divided into nonoverlapping 2-noded elements. Therefore, it holds that:

$$
\int(\bullet) d z=\sum_{e=1}^{N_{e}} \int_{e}(\bullet) d z,
$$

where $N_{e}$ is the number of elements.

The trapezium rule is then used to spatially integrate both the conservation of mass and the momentum equation from node 1 to node 2 . Note that the flows are here defined conventionally meaning that both flows within the element are directed from node 1 to node 2 . The integration over the element domain $e$, using the trapezium rule yields:

$$
\begin{array}{r}
\int_{e} C_{A} \frac{\partial p}{\partial t} d z \approx \frac{\Delta z_{e}}{2}\left(C_{A, 1} \frac{\partial p_{1}}{\partial t}+C_{A, 2} \frac{\partial p_{2}}{\partial t}\right), \\
\int_{e}\left(\frac{\partial p}{\partial z}\right) d z \approx\left(p_{2}-p_{1}\right), \\
\int_{e} \frac{\rho}{A} \frac{\partial q}{\partial t} d z \approx \frac{\Delta z_{e}}{2}\left(\frac{\rho}{A_{1}} \frac{\partial q_{1}^{c}}{\partial t}+\frac{\rho}{A_{2}} \frac{\partial q_{2}^{c}}{\partial t}\right), \\
\int_{e} \frac{\partial q}{\partial z} d z=\left(q_{2}^{c}-q_{1}^{c}\right), \quad \int_{e} h d z \approx \frac{\Delta z_{e}}{2}\left(h_{1}+h_{2}\right), \\
h_{i}=\left\{-\frac{8 \mu \pi q}{A^{2}}-\frac{\rho}{A} \frac{\partial}{\partial z}\left(\frac{q^{2}}{A}\right)\right\}_{i} .
\end{array}
$$

Here, $\Delta z_{e}$ is the element length and the superscript $c$ denotes the use of the conventional definition of flows, note that at this point thus different from the definition used during our numerical implementation. In matrix form, (A6) becomes:

$$
\begin{array}{r}
{\left[\begin{array}{c}
C_{A, 1} \frac{\Delta z_{e}}{2} \\
C_{A, 2} \frac{\Delta z_{e}}{2}
\end{array}\right]^{T} \frac{\partial \underline{p}}{\partial t}+\left[\begin{array}{l}
-1 \\
+1
\end{array}\right]^{T} \underline{q}_{e}^{c}=0,} \\
{\left[\begin{array}{c}
\frac{\rho}{A_{1}} \frac{\Delta z_{e}}{2} \\
\frac{\rho}{A_{1}} \frac{\Delta z_{e}}{2}
\end{array}\right]^{T} \frac{\partial q_{e}^{c}}{\partial t}+\left[\begin{array}{l}
-1 \\
+1
\end{array}\right]^{T} \underline{p}_{e}=\left(h_{1}+h_{2}\right) \frac{\Delta z_{e}}{2},}
\end{array}
$$

where $\underline{p}_{e}=\left[p_{1}, p_{2}\right]^{T}$ and $\underline{q}_{e}^{c}=\left[q_{1}^{c}, q_{2}^{c}\right]^{T}$ contain nodal point pressures and flows of the element, respectively. We now apply a second-order backward differentiation scheme for the time derivatives with time step $\Delta t$. Now, we can rewrite (A7) in the following matrix notation:

$$
\mathbf{F}_{\mathbf{e}} \underline{p}^{t+\Delta t}+\mathbf{G}_{e}^{c} \underline{q}_{e}^{c, t+\Delta t}=\underline{h}_{e}
$$

where:

$$
\mathbf{F}_{\mathbf{e}}=\left[\begin{array}{cc}
\frac{3}{2 \Delta t} \frac{\Delta z}{2} C_{A, 1}^{t} & \frac{3}{2 \Delta t} \frac{\Delta z}{2} C_{A, 2}^{t} \\
-1 & +1
\end{array}\right],
$$




$$
\begin{aligned}
& \mathbf{G}_{\mathbf{e}}^{\mathbf{c}}=\left[\begin{array}{cc}
-1 & -1 \\
\frac{3}{2 \Delta t} \frac{\Delta z}{2} \frac{\rho}{A_{1}^{t}} & -\frac{3}{2 \Delta t} \frac{\Delta z}{2} \frac{\rho}{A_{2}^{t}}
\end{array}\right] \\
& \underline{h_{e}}=\left[\begin{array}{c}
0 \\
\left(h_{1}^{t}+h_{2}^{t}\right) \frac{\Delta z}{2}
\end{array}\right]+\left[\begin{array}{c}
\frac{\Delta z}{2}\left(\frac{2}{\Delta t} C_{A, 1}^{t} p_{1}^{t}-\frac{1}{2 \Delta t} C_{A, 1}^{t} p_{1}^{t-\Delta t}\right) \\
\frac{\Delta z}{2}\left(\frac{2}{\Delta t} \frac{\rho}{A_{1}^{t}} q_{1}^{t}-\frac{1}{2 \Delta t} \frac{\rho}{A_{1}^{t}} q_{1}^{t-\Delta t}\right)
\end{array}\right]+\left[\begin{array}{c}
\frac{\Delta z}{2}\left(\frac{2}{\Delta t} C_{A, 2}^{t} p_{2}^{t}-\frac{1}{2 \Delta t} C_{A, 2}^{t} p_{2}^{t-\Delta t}\right) \\
\frac{\Delta z}{2}\left(\frac{2}{\Delta t} \frac{\rho}{A_{2}^{t}} q_{2}^{t}-\frac{1}{2 \Delta t} \frac{\rho}{A_{2}^{t}} q_{2}^{t-\Delta t}\right)
\end{array}\right] .
\end{aligned}
$$

Next, we make a switch to the discretization as depicted in Figure 2, where both flows are defined inwards, that is, $q_{1}=q_{1}^{c}$ and $q_{2}=-q_{2}^{c}$. Now, we can rewrite (A8) in the following matrix notation form:

$$
\mathbf{F}_{\mathbf{e}} \underline{p}^{t+\Delta t}+\mathbf{G}_{e} \underline{q}_{e}^{t+\Delta t}=\underline{h}_{e},
$$

with:

$$
\mathbf{G}_{\mathbf{e}}=\left[\begin{array}{ll}
G_{e, 11}^{c} & -G_{e, 12}^{c} \\
G_{e, 21}^{c} & -G_{e, 22}^{c}
\end{array}\right], \quad \underline{q}_{e}=\left[\begin{array}{c}
q_{1}^{c} \\
-q_{2}^{c}
\end{array}\right]=\left[\begin{array}{l}
q_{1} \\
q_{2}
\end{array}\right]
$$

After separation of matrix $G_{e}$ from flow column $\underline{q}_{e}^{t+\Delta t}$, the 1D line element equations finally read:

$$
\left[-\mathbf{G}_{e}^{-1} \mathbf{F}_{e}\right] \underline{p}_{e}^{t+\Delta t}=\underline{q}_{e}^{t+\Delta t}+\left[-\mathbf{G}_{e}^{-1} \underline{h}_{e}\right],
$$

which is now cast into the same general form:

$$
\mathbf{K}_{e}^{t, 1 D} \underline{p}_{e}^{t+\Delta t}=\underline{q}_{e}^{t+\Delta t}+\underline{f}_{e}^{t, 1 D}
$$

\section{APPENDIX B : ZERO-DIMENSIONAL WINDKESSEL ELEMENT EQUATIONS}

The three elements in Figure 3 are described by two resistors and one compliance. In matrix form, the equations for these elements become:

$$
\begin{aligned}
& 1 / R_{1}\left[\begin{array}{cc}
1 & -1 \\
-1 & 1
\end{array}\right]\left[\begin{array}{l}
p_{1} \\
p_{2}
\end{array}\right]=\left[\begin{array}{l}
q_{1} \\
q_{2}
\end{array}\right], \\
& 1 / R_{2}\left[\begin{array}{cc}
1 & -1 \\
-1 & 1
\end{array}\right]\left[\begin{array}{l}
p_{2} \\
p_{3}
\end{array}\right]=\left[\begin{array}{l}
q_{3} \\
q_{4}
\end{array}\right], \\
& C\left[\begin{array}{cc}
1 & -1 \\
-1 & 1
\end{array}\right]\left[\begin{array}{l}
\frac{\partial p_{2}}{\partial t} \\
\frac{\partial p_{4}}{\partial t}
\end{array}\right]=\left[\begin{array}{l}
q_{5} \\
q_{6}
\end{array}\right] .
\end{aligned}
$$

These element can be assembled to:

with:

$$
\mathbf{C}_{e} \frac{\partial \underline{p}}{\partial t}+\mathbf{R}_{e}^{r} \underline{p}_{e}=\underline{q}_{e}
$$

$$
\begin{gathered}
\mathbf{C}_{e}=\left[\begin{array}{cccc}
0 & 0 & 0 & 0 \\
0 & C & -C & 0 \\
0 & 0 & 0 & 0 \\
0 & -C & C & 0
\end{array}\right], \\
\mathbf{R}_{e}^{r}=\left[\begin{array}{cccc}
\frac{1}{Z} & -\frac{1}{Z} & 0 & 0 \\
-\frac{1}{Z} & \frac{1}{Z}+\frac{1}{R} & 0 & -\frac{1}{R} \\
0 & -\frac{1}{R} & 0 & \frac{1}{R} \\
0 & 0 & 0 & 0
\end{array}\right],
\end{gathered}
$$


where $\underline{p}_{e}=\left[p_{1}, p_{2}, p_{3}, p_{4}\right]^{T}$ and $\underline{q}_{e}=\left[q_{1}, q_{2}+q_{3}+q_{5}, q_{4}, q_{6}\right]^{T}$.

Application of the second-order backward differentiation scheme on (B4) results in:

$$
\left(\frac{3}{2 \Delta t} \mathbf{C}_{e}+\mathbf{R}_{e}^{r}\right) \underline{p}_{e}^{t+\Delta t}=\left(\mathbf{C}_{e}\left(-\frac{-2}{\Delta t} p_{e}^{t}-\frac{1}{2 \Delta t} \underline{p}_{e}^{t-\Delta t}\right)\right)+\underline{q}_{e}^{t+\Delta t}
$$

which is now cast into the same general form:

$$
\mathbf{K}_{e}^{t, 0 D} \underline{p}_{e}^{t+\Delta t}=\underline{f}_{e}^{t, 0 D}+\underline{q}_{e}^{t+\Delta t}
$$

\section{APPENDIX C : NEWTON-RAPHSON LINEARIZATION}

The linearization of (14) is performed using the Newton-Raphson method. This method is used to step forward in time and uses the current time step to estimate the pressure of the next time step. At time step $t$, (14) gives:

$$
p_{i}^{t}-p_{4}^{t}=\frac{1}{2} \rho\left(\frac{\left(q_{r}^{t}\right)^{2}}{\left(A_{r}^{t}\right)^{2}}-\frac{\left(q_{i}^{t}\right)^{2}}{\left(A_{i}^{t}\right)^{2}}\right) .
$$

The right-hand side can then be written as a function with variable $q_{i}$, which results in:

$$
p_{i}^{t}-p_{4}^{t}=f\left(q_{i}^{t}\right) .
$$

If we use this equation to step forward in time, this results in:

$$
p_{i}^{t+\Delta t}-p_{4}^{t+\Delta t}=f\left(q_{i}^{t+\Delta t}\right)
$$

with $\Delta t$ the time step. We now want to apply the Newton-Raphson method for the right-hand side, which uses the previous time step and estimates the difference with the next time step:

$$
p_{i}^{t+\Delta t}-p_{4}^{t+\Delta t}=f\left(q_{i}^{t}\right)+\frac{\partial f\left(q_{i}^{t}\right)}{\partial q_{i}^{t}}\left(q_{i}^{t+\Delta t}-q_{i}^{t}\right) .
$$

Here, the higher order terms are omitted. Using this Newton-Raphson method, (C1) can finally be rewritten to (15):

$$
p_{i}^{t+\Delta t}-p_{4}^{t+\Delta t}=-\rho \frac{q_{i}^{t}}{\left(A_{i}^{t}\right)^{2}} q_{i}^{t+\Delta t}+\frac{\rho}{2} \frac{\left(q_{i}^{t}\right)^{2}}{\left(A_{i}^{t}\right)^{2}}+\frac{\rho}{2} \frac{\left(q_{r}^{t}\right)^{2}}{\left(A_{r}^{t}\right)^{2}},
$$

with $i=1,2,3$. 